\title{
Target optimization in transcranial direct current stimulation
}

\author{
Rosalind J. Sadleir ${ }^{1,2}{ }^{*}$, Tracy D. Vannorsdall ${ }^{3}$, David J. Schretlen ${ }^{3,4}$ and Barry Gordon ${ }^{5,6}$ \\ 1 J. Crayton Pruitt Family Department of Biomedical Engineering, University of Florida, Gainesville, FL, USA \\ ${ }^{2}$ Department of Biomedical Engineering, Kyung Hee University, Seoul, South Korea \\ ${ }^{3}$ Department of Psychiatry and Behavioral Sciences, The Johns Hopkins University School of Medicine, Baltimore, MD, USA \\ ${ }^{4}$ Russell H. Morgan Department of Radiology and Radiological Science, The Johns Hopkins University School of Medicine, Baltimore, MD, USA \\ ${ }^{5}$ Department of Neurology, Cognitive Neurology/Neuropsychology, The Johns Hopkins University School of Medicine, Baltimore, MD, USA \\ ${ }^{6}$ Department of Cognitive Science, The Johns Hopkins University, Baltimore, MD, USA
}

\section{Edited by:}

Andre R. Brunoni, Universidade de São Paulo, Brazil

\section{Reviewed by:}

Abhishek Datta, Soterix Medical, USA

Pedro Shiozawa, Santa Casa de

Misericórdia de São Paulo, Brazil

*Correspondence:

Rosalind J. Sadleir, J. Crayton Pruitt

Family Department of Biomedical

Engineering, University of Florida,

Box 116131, Gainesville, FL

32611-6131, USA

e-mail:sadleir@ufl.edu
Transcranial direct current stimulation (tDCS) is an emerging neuromodulation therapy that has been experimentally determined to affect a wide range of behaviors and diseases ranging from motor, cognitive, and memory processes to depression and pain syndromes. The effects of tDCS may be inhibitory or excitatory, depending on the relative polarities of electrodes and their proximity to different brain structures. This distinction is believed to relate to the interaction of current flow with activation thresholds of different neural complexes. tDCS currents are typically applied via a single pair of large electrodes, with one (the active electrode) sited close to brain structures associated with targeted processes. To efficiently direct current toward the areas presumed related to these effects, we devised a method of steering current toward a selected area by reference to a 19-electrode montage applied to a high-resolution finite element model of the head. We used a non-linear optimization procedure to maximize mean current densities inside the left inferior frontal gyrus (IFG), while simultaneously restricting overall current, and median current densities within the accumbens. We found that a distributed current pattern could be found that would indeed direct current toward the IFG in this way, and compared it to other candidate 2-electrode configurations. Further, we found a combination of four anterior-posterior electrodes could direct current densities to the accumbens. We conclude that a similar method using multiple electrodes may be a useful means of directing current toward or away from specific brain regions and also of reducing tDCS side effects.

Keywords: tDCS, neuroplasticity, finite element model, optimization

\section{INTRODUCTION}

Transcranial direct current stimulation (tDCS) is an emerging method for modulation of brain function. Applications have been widely tested in experimental scenarios of motor, semantic, and attention processes (Nitsche et al., 2008). Other recent experimental uses include therapy for depression and hallucinations in schizophrenia (Brunelin et al., 2012; Loo et al., 2012).

The mechanism of tDCS is believed to arise through a modulation of baseline cortical excitability, caused by shifts in resting membrane potentials in regions experiencing current flow (Brunoni et al., 2012). The effects of tDCS depend on the relative polarity of electrodes. In general, anodal tDCS (where the active electrode is more positive than the reference electrode) has excitatory effects, and cathodal tDCS has inhibitory effects (Nitsche and Paulus, 2000). This has been substantiated in numerous experiments. For example studies of tDCS in cognitive tasks found that anodal tDCS delivered over the dorsolateral prefrontal cortex facilitated visual working memory (Fregni et al., 2005) and cathodal stimulation impaired short-term auditory memory performance (Elmer et al., 2009). Application of tDCS may, in turn affect the manifestations of neuropsychiatric conditions, including autism, depression, migraine, and schizophrenia, as baseline cortical excitability is characteristic of these conditions (Brunoni et al., 2012).

Little is known about the exact current flow patterns elicited by tDCS. Although methods using MRI scanners exist for measuring intracranial current flow (Scott et al., 1991), they are not conveniently applied because of the need for subject repositioning. Detailed models of current flow have therefore been created using finite element modeling in lieu of actual current measurement (Wagner et al., 2007; Datta et al., 2009; Sadleir et al., 2010). Though modeling is informative, there is still no clear mechanism linking current direction, current distribution, and observed experimental effects.

If it is possible to direct current toward or away from specific brain areas, the mechanisms, and structures responsible for the observed effects of tDCS may become clear. The ability to control current distribution throughout the brain may also provide a deeper understanding of general neural circuitry and networks. To best determine the stimulation parameters required to target different brain areas, we must refer to a complete electrical model of the head. This approach is natural because the paths 
taken by transcranial currents are defined by head geometry and conductivity, as well as electrode shape and location.

In this study, we performed tests using a non-linear optimization technique to determine if current densities in brain structures could be shaped. We investigated three scenarios: one in which we wished to target cortical structures and to avoid the accumbens; a second in which we wished to target the region of the accumbens (left and right) with no constraint on regions to be avoided; and a third in which the accumbens was targeted, but the left inferior frontal gyrus (IFG) was avoided.

Other authors have used related optimization approaches (Im et al., 2008; Dmochowski et al., 2011; Park et al., 2011). While Im et al. (2008) used a evolution strategy approach to find optimal two-electrode locations from which to target a nominated brain area, a more recent work from their group used fixed anterior and posterior electrode location and a simplex algorithm to determine the appropriate current amplitudes needed to apply maximal currents (Park et al., 2011). Similarly, Dmochowski et al. (2011) used a fixed 64-electrode array and a variety of optimization approaches to determine current amplitudes needed to create maximal currents in a nominated cortical area.

Our methods use a general non-linear algorithm, which allows for flexible and general constraints to be applied. Dmochowski et al. (2011) used a similar approach. We used a linear basis for our computations comprising calculations of current flows between individual electrodes and a reference ground plane, whereas Park et al. (2011) and Dmochowski et al. (2011) used pairs of modeled electrodes to compute test intracranial current patterns. Our approach led to the implicit option to include extracranial electrodes. Normally the sum of all currents flowing into and out of the head should be zero. However, in part of the work presented here we have calculated optimal current flows through electrodes without this constraint. Any uncompensated current flowing through scalp electrodes after optimization can then be accounted for in real experiments by attaching an extracranial electrode to complete the circuit and supply the remaining current. As in Dmochowski et al. (2011) we used a general non-linear algorithm that allowed the inclusion of both target and avoidance areas. In contrast to their approach, we have explicitly specified avoidance areas rather than seeking to minimize current densities in all regions outside the target. Our method used large electrodes similar to those currently used in tDCS studies. Use of large electrodes avoids the risk of applying large currents to the skin, an effect that can lead to superficial burning. Finally, the model used as the base for our computations included white matter anisotropy. This more realistic model potentially facilitates better current localization and helped us discern an intriguing anatomical asymmetry in our test model.

\section{MATERIALS AND METHODS}

In the following sections we detail our electrical head model and the constructs and calculations used in optimization procedures.

\section{TISSUE SEGMENTATION AND CONDUCTIVITY ASSIGNMENTS}

We used the "Re-sliced Adam" (RA) dataset from the DTI White Matter atlas repository housed at the Johns Hopkins Medical Institutes (http://cmrm.med.jhmi.edu/). The RA model is a single subject atlas with a resolution of $1 \times 1 \times 1 \mathrm{~mm}^{3}$ and includes white matter anisotropy vectors and $\mathrm{T} 1$ weighted (MPRAGE) MR images (Wakana et al., 2004). Segmentation was performed using both automatic classification and manual comparison with an anatomical atlas (Rubin and Safdieh, 2007). Non-brain data were segmented manually using ScanIP (Simpleware, Exeter, UK) software into 10 tissue types: cancelous bone, cortical bone, blood, cerebrospinal fluid (CSF), sclera, fat, muscle, brain, and skin. The original model did not include slices above the superior limit of the cortex. Therefore, to include the crown of the head, we extended the model by adding 12 slices ( $12 \mathrm{~mm}$ height) to the superior portion of the model, completing the head with CSF, cortical bone, and scalp materials. The brain tissue itself was further segmented automatically using FreeSurfer 5.0.0 (Cambridge, MA, USA) software into white matter and gray matter; and then subclassified into many cortical and deep brain structures. Specific target areas used in this study - the IFG, angular gyrus (AG), and dorsolateral prefrontal cortex (DLPFC) - were isolated using manually, referring anatomical atlas information.

Conductivity values were assigned to each tissue, chosen from measurements reported below $1 \mathrm{kHz}$. Table 1 lists the sources for conductivities.

White matter was assumed anisotropic. We distinguished between conductivities of cancelous and cortical bone because of the large electrical property differences between these tissues (Akhtari et al., 2000, 2002; Sadleir and Argibay, 2007).

\section{FINITE ELEMENT MODELING}

The model solved the Laplace equation

$\nabla \cdot(\sigma(x, y, z) \nabla \phi)=0$

on the domain $\Omega$ (the head), subject to

$\sigma \frac{\partial \phi}{d \mathbf{n}}=j$ and $\phi_{\text {base }}=0$

Table 1 | Conductivities assigned to tissues in our model.

\begin{tabular}{lll}
\hline Compartment & Conductivity (S/m) & Reference \\
\hline Air & 0 & - \\
Skin & $4.3 \times 10^{-1}$ & Holdefer et al. (2006) \\
Cerebrospinal fluid & $1.8 \times 10^{0}$ & Baumann et al. (1997) \\
Sclera & $5.0 \times 10^{-1}$ & Gabriel et al. (1996) \\
Cortical bone & $5.52 \times 10^{-3}$ & Akhtari et al. (2002) \\
Cancelous bone & $21.4 \times 10^{-3}$ & Akhtari et al. (2002) \\
Muscle* & $1.6 \times 10^{-1}$ & Geddes and Baker (1967) \\
Fat & $2.5 \times 10^{-2}$ & Gabriel et al. (1996) \\
Blood & $6.7 \times 10^{-1}$ & Geddes and Baker (1967) \\
White matter* & $1.2 \times 10^{-1}$ (trans.) & Geddes and Baker (1967) \\
& $1.2 \times 10^{-0}$ (long.) & \\
Gray matter & $1.0 \times 10^{-1}$ & Gabriel et al. (1996)
\end{tabular}

Values were chosen from available low frequency $(<1 \mathrm{kHz})$ data in the literature. Compartments marked with an asterisk were anisotropic. The isotropic conductivity assigned to muscle was calculated according to the formula $\sigma^{*}=\left(\sigma_{l} \sigma_{t}\right)$ where $\sigma_{l}$ is longitudinal and $\sigma_{t}$ is transverse measured conductivity. 
on the surface of the domain $d \Omega$. Here, $\sigma(x, y, z)$ is the conductivity distribution within the head, $\phi$ is the voltage distribution, $j$ is the surface current density, and $\mathbf{n}$ is a vector normal to the surface. The quantity $j$ was only non-zero on electrodes. The voltage on the base plane (the caudal slice) of the model ( $\phi$ base $)$ was set to zero.

The segmented phantom was converted into a quadratic tetrahedral finite element model containing $\sim 18$ million elements. In each white matter voxel, the anisotropic conductivity tensor was calculated as

$\boldsymbol{D}_{W}=\boldsymbol{A}^{T} \boldsymbol{D}^{*}{ }_{W} \boldsymbol{A}$

where

$$
\boldsymbol{D}^{*}{ }_{W}=\left[\begin{array}{ccc}
\sigma_{l} & 0 & 0 \\
0 & \sigma_{t} & 0 \\
0 & 0 & \sigma_{t}
\end{array}\right] \text { and } \boldsymbol{A}=\boldsymbol{R}_{z} \boldsymbol{R}_{y} \boldsymbol{R}_{x} . \boldsymbol{R}_{z}, \boldsymbol{R}_{y} \text {, and } \boldsymbol{R}_{x} \text { are }
$$

rotation matrices about the $z, y$, and $x$ axes, respectively. In isotropic voxels, $\mathbf{D}$ was a diagonal matrix with all entries equal to the local isotropic conductivity value.

Computations of finite element model matrix equations and boundary conditions were implemented in $\mathrm{C}$ and solved using the preconditioned conjugate gradient method.

\section{Electrode assignment and definition}

Transcranial direct current stimulation current is normally introduced via a pair of large $\left(\sim 35 \mathrm{~cm}^{2}\right)$ saline/sponge electrodes. One (the active electrode) is sited close to brain regions presumed involved in target processes. The other (the reference electrode) is placed elsewhere on the head or body. For this study, we defined a montage of $\mathrm{NE}=19$ electrodes (Figure 1). The electrodes were selected from standard 10-20 EEG locations. Each electrode had an area of $\sim 22 \mathrm{~cm}^{2}$. Use of large electrodes reduces the risk that superficial burns will result from current application.

\section{Boundary conditions}

The base data used in the optimization procedure consisted of voltage data calculated between each electrode and a ground plane situated at the base of the model (Figure 2). In calculating the voltage data for each isolated electrode in turn, we simulated a total current of $1 \mathrm{~mA}$ injected into the head. Use of this single electrode arrangement allowed us to include the possibility that extracranial electrodes could be included (simply by allowing the sum of currents applied to the model to have a net non-zero value, implying that the extra current flowed through the neck and to an electrode located away from the head.

A

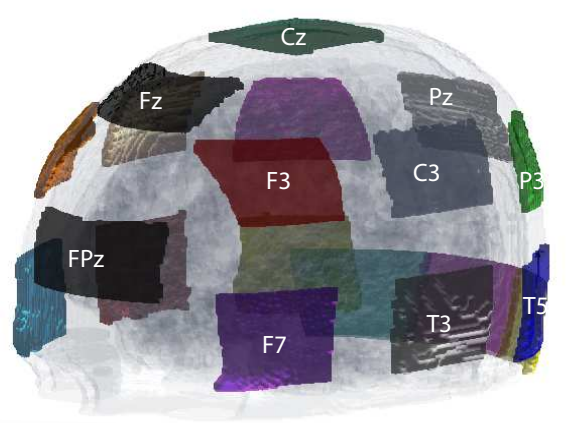

B

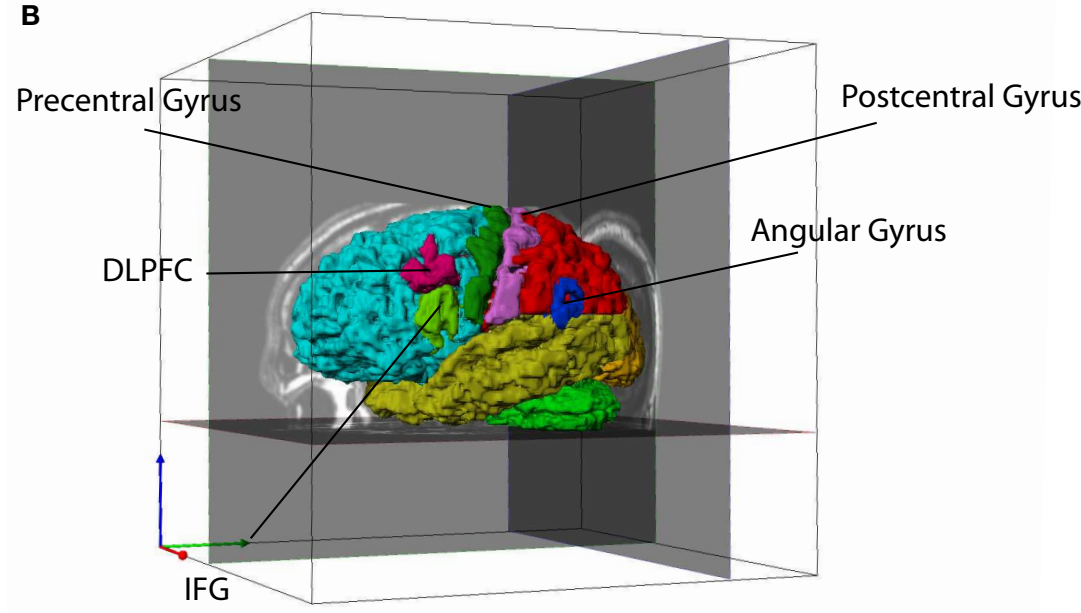

FIGURE 1 | Electrode montage and tissue segmentation. (A) Left frontal view of electrode montage shown on a transparent head model. There were 19 electrodes in total, not all of which are shown. (B) Segmented cortical structures, showing frontal (aqua), parietal (red), temporal (yellow), occipital (orange) lobes, cereballar gray matter (green), angular gyrus (AG, blue), dorsolateral prefrontal cortex (DLPFC, cyan), inferior frontal gyrus (IFG, light green), precentral cortex (dark green), and postcentral cortex (pink). 


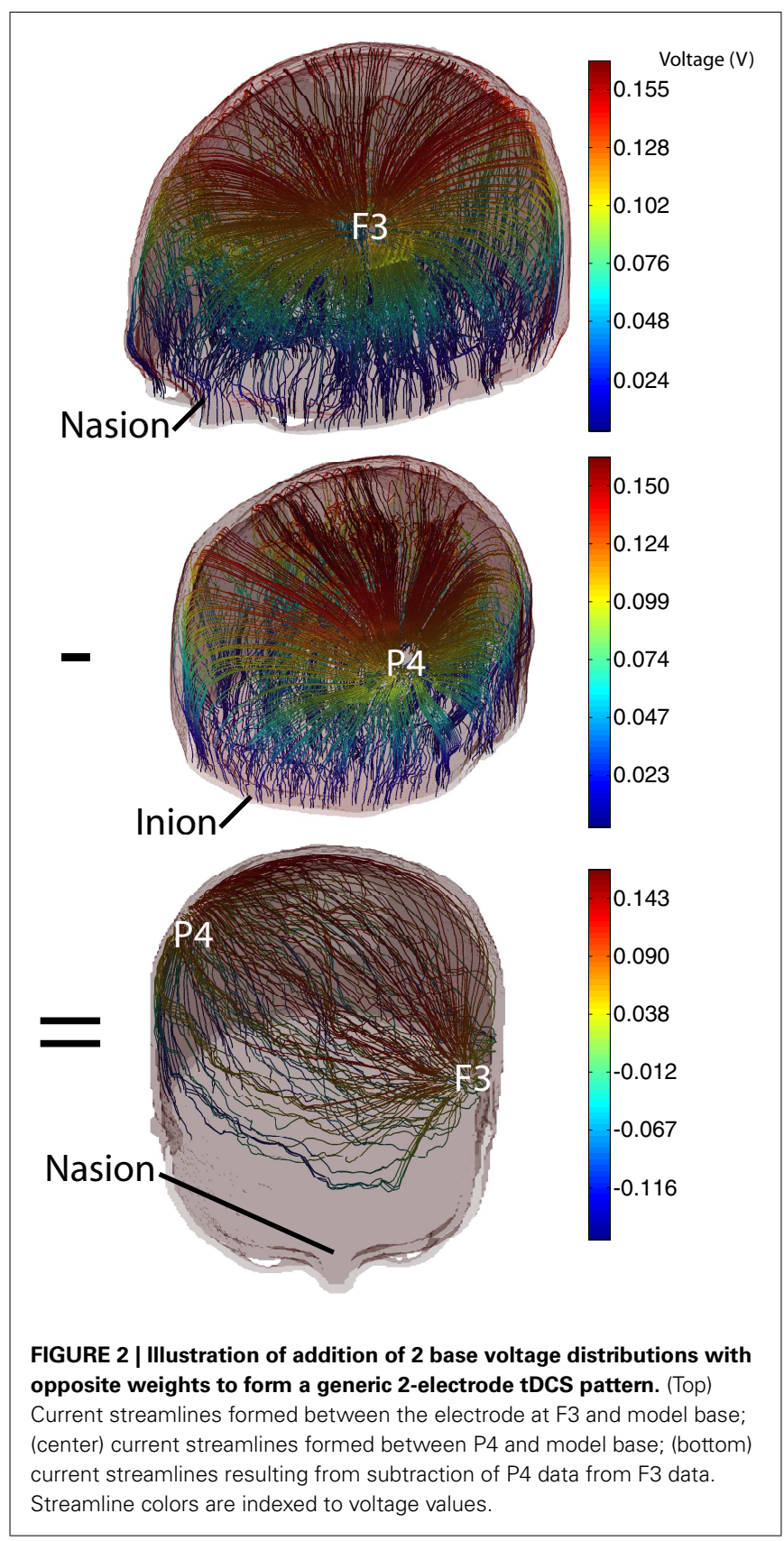

\section{DATA COMPUTATION}

Voltage distributions for a particular electrode combination were computed using the principle of superposition by summing the weighted basis data set as

$V_{X}=X_{1} V_{1}+X_{2} V_{2}+\cdots+X_{N E} V_{N E}$

where $\boldsymbol{X}=\left[X_{1} X_{2} \ldots X_{N E}\right]$ was a vector of weighting factors for each voltage data set, $V_{1} \ldots N E$ were the basis data sets, and $V_{X}$ was the resulting voltage. Figure 2 shows the result of weighted summation of voltage basis data using electrodes F3 and P4. The top and center panels of Figure 2 show individual voltage basis data for electrodes F3 and P4, and the lower panel shows the result of adding data for electrode $\mathrm{F} 3$ (weight $1 \mathrm{~mA}$ ) to data for P3 (weight $-1 \mathrm{~mA}$ ). The total current magnitude injected into the head was computed as

$C_{\text {total }}=\frac{1}{2} \sum_{i=1}^{N E}\left|X_{i}\right|$

The current density $\mathbf{J}$ in each voxel $k$ was calculated as

$J_{k}=-D_{k} \nabla \phi$

where $\nabla \phi$ is the local voltage gradient.

Current density norms $J$ were calculated within each voxel from individual vector components as

$J=\left(J_{x}^{2}+J_{y}^{2}+J_{z}^{2}\right)^{1 / 2}$

This distribution was then used to compute mean or median current densities within regions of interest.

\section{OPTIMIZATION PROCEDURE}

We used the interior point optimization method to calculate the optimal electrode currents. The interior point algorithm (Waltz et al., 2006) solves a general non-linear minimization problem subject to linear and non-linear constraints. Other methods for solving such problems include sequential quadratic programming methods (Bonnans et al., 2006) and simulated annealing (Kirkpatrick et al., 1983).

Our interior point optimization algorithm was implemented in the MATLAB (Natick, MA, USA) function fmincon to solve.

$$
\begin{aligned}
& \max _{X}\left[\operatorname{mean}\left(J_{\text {target }}(\boldsymbol{X})\right)\right], \\
& \text { such that }\left\{\begin{array}{l}
\sum_{i=1}^{N E} X_{i}=0 \\
\text { mean }\left(J_{\text {avoid }}(\boldsymbol{X})\right)<J_{\max } \\
\sum_{i=1}^{N E}\left|X_{i}\right|>C_{\min } \\
\sum_{i=1}^{N E}\left|X_{i}\right|<C_{\max } \\
\text { mean }\left(J_{\text {target }}(\boldsymbol{X})\right) \geq r \text { mean }\left(J_{\text {avoid }}(\boldsymbol{X})\right)
\end{array}\right.
\end{aligned}
$$

Here, $\boldsymbol{X}$ is the vector consisting of coefficients denoting the stimulus intensity to be delivered to each electrode, and $J$ refers to the current density norm within a brain structure (a target region or a region to avoid). The quantity $\max _{X}\left[\operatorname{mean}\left(J_{\text {target }}(\boldsymbol{X})\right)\right]$ is the objective function. The optimization is subject to the constraints that the total current injected into the brain is zero (constraint 1 ), the mean $J$ delivered to the "avoid" region is less than a prescribed maximum value $\left(J_{\max }\right.$, constraint 2$)$, the total absolute delivered current is above a set threshold $\left(C_{\min }\right.$, constraint 3 ) and below another threshold $\left(C_{\max }\right.$, constraint 4 ), and the mean $J$ in the target region is at least $r$ times the mean current density in the avoid region, where $r$ is a dimensionless constant (constraint 5). Only constraint 4 is essential. For example, if constraint 1 is not 


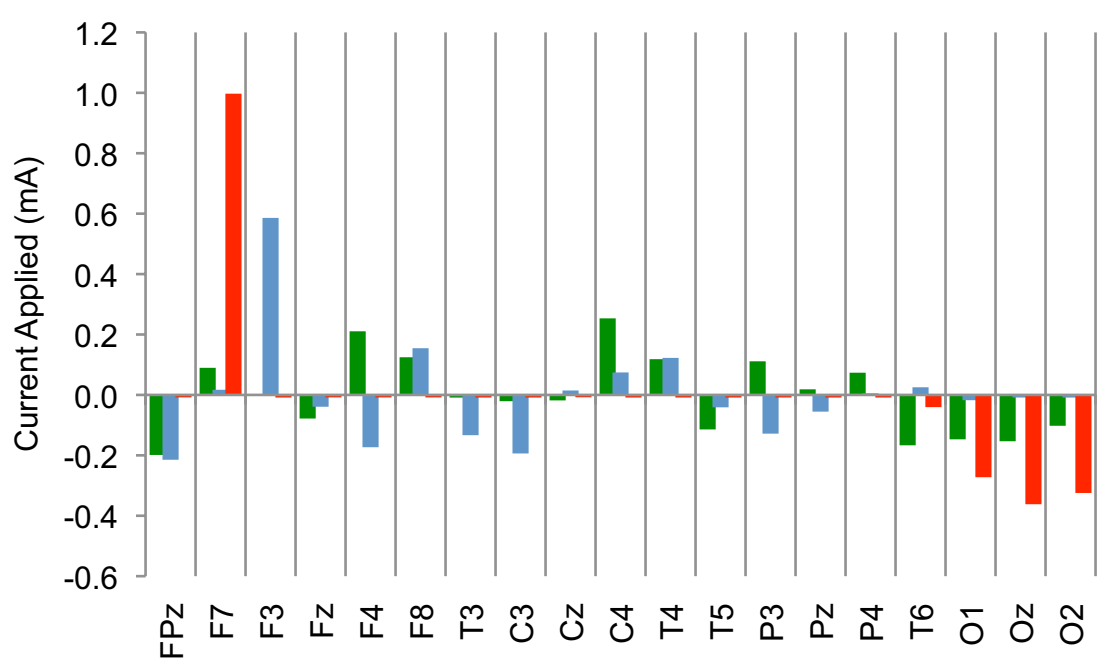

\begin{tabular}{cccccccccc}
\hline Electrode & \multicolumn{10}{c}{ Electrode $(\mathrm{A}-\mathrm{P})$} \\
Problem & FPz & F7 & F3 & Fz & F4 & F8 & T3 & C3 & Cz \\
1 & -0.215 & 0.017 & 0.586 & -0.039 & -0.173 & 0.155 & -0.133 & -0.194 & 0.015 \\
2 & 0.001 & 0.997 & 0.000 & 0.001 & 0.000 & 0.000 & 0.000 & 0.000 & 0.001 \\
3 & -0.199 & 0.090 & 0.000 & -0.078 & 0.211 & 0.125 & -0.001 & -0.021 & -0.018 \\
\hline 1 & 0.123 & -0.041 & -0.128 & -0.055 & 0.005 & 0.025 & -0.017 & 0.000 & -0.004 \\
2 & 0.000 & 0.000 & 0.000 & 0.000 & 0.000 & -0.040 & -0.272 & -0.362 & -0.325 \\
3 & 0.118 & -0.114 & 0.111 & 0.019 & 0.074 & -0.167 & -0.147 & -0.153 & -0.102
\end{tabular}

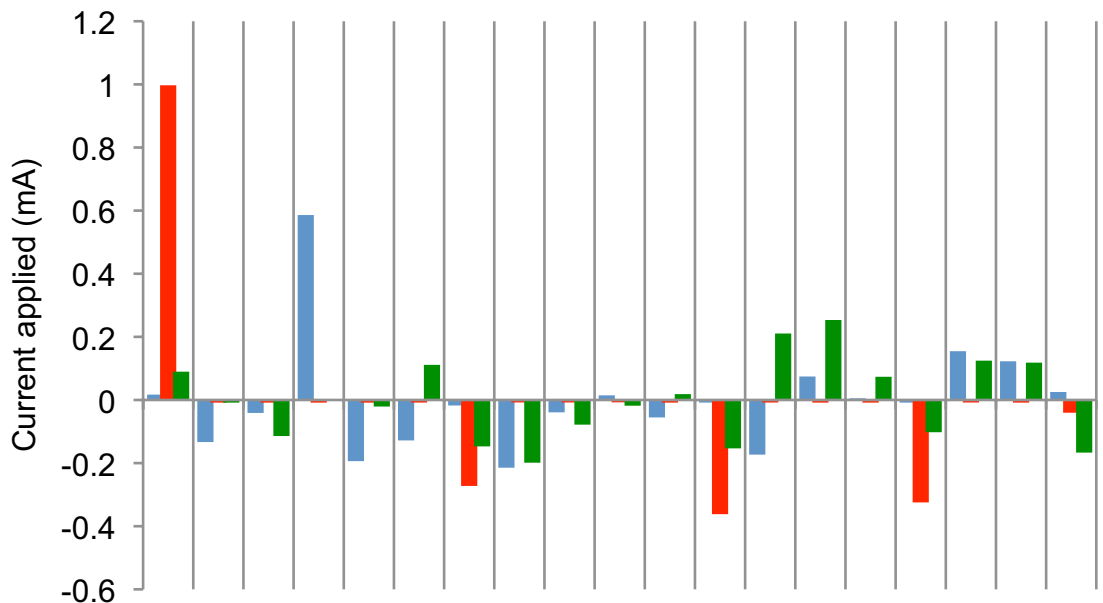

Problem 1

- Problem 2

- Problem 3

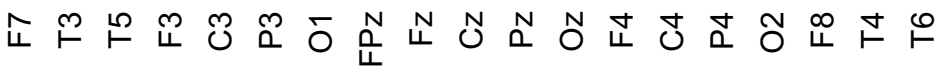

\begin{tabular}{|c|c|c|c|c|c|c|c|c|c|c|}
\hline \multicolumn{11}{|c|}{ Electrode (L-R) } \\
\hline Problem & F7 & T3 & T5 & F3 & $\mathrm{C} 3$ & P3 & $\mathrm{O} 1$ & $\mathrm{FPz}$ & $\mathrm{Fz}$ & $\mathrm{Cz}$ \\
\hline 1 & 0.017 & -0.133 & -0.041 & 0.586 & -0.194 & -0.128 & -0.017 & -0.215 & -0.039 & 0.015 \\
\hline 2 & 0.997 & 0.000 & 0.000 & 0.000 & 0.000 & 0.000 & -0.272 & 0.001 & 0.001 & 0.001 \\
\hline 3 & 0.090 & -0.001 & -0.114 & 0.000 & -0.021 & 0.111 & -0.147 & -0.199 & -0.078 & -0.018 \\
\hline & $\mathrm{Pz}$ & $\mathrm{Oz}$ & F4 & C4 & P4 & $\mathrm{O} 2$ & F8 & T4 & T6 & \\
\hline 1 & -0.055 & 0.000 & -0.173 & 0.074 & 0.005 & -0.004 & 0.155 & 0.123 & 0.025 & \\
\hline 2 & 0.000 & -0.362 & 0.000 & 0.000 & 0.000 & -0.325 & 0.000 & 0.000 & -0.040 & \\
\hline 3 & 0.019 & -0.153 & 0.211 & 0.253 & 0.074 & -0.102 & 0.125 & 0.118 & -0.167 & \\
\hline
\end{tabular}

FIGURE 3 | Optimized current weights (in $\mathrm{mA}$ ) found in the three problems, shown in graphical and tabular format. Weights are displayed in (top) Anterior-Posterior and (bottom) Left-Right arrangements. 
applied, any unbalanced flow of current through the ground plane may be considered as flow to or from an extracranial electrode, such as those used in several previous studies (Cogiamanian et al., 2007; Monti et al., 2008; Priori et al., 2008). We may consider other constraints, such as a limit on the maximum skin $J$.

\section{Termination criteria}

The optimization procedure was terminated if more than 100 iterations were required, if the relative step size of any iteration was below 1 part in $10^{10}$ or if the gradient estimate was below 1 part in $10^{3}$. A feasible solution was considered achieved if the maximum constraint violation was smaller than 1 part in $10^{10}$.

\section{Mean and median current density values}

Although we have previously (Sadleir et al., 2010) quoted median current densities as best representative of distributions, and have observed that the current density distributions are approximately log-normal, there is no analytical method to associate the median of sums and the sum of medians for log-normal distributions (Limpert et al., 2001). This limitation prevents us from associating median current densities in individual base current distributions with the median of their sum. Consequently, the gradient of the objective function cannot be computed, except numerically. Numerical gradient estimation requires many extra function estimations and greatly slows the optimization algorithm. We therefore estimated the gradient of the objective function by computing the mean $J$ created in the target region for each of the 19 candidate patterns. This approach does not produce an exact gradient, but the sum of weighted mean current densities is greater than or equal to actual mean $J$ values, that is

$$
\begin{aligned}
& J=|\boldsymbol{J}| \leq X_{1}\left|\boldsymbol{J}_{1}\right|+X_{2}\left|\boldsymbol{J}_{2}\right|+\cdots+X_{N E}\left|\boldsymbol{J}_{N E}\right| \\
& J_{\text {target,avoid }}=\left|\boldsymbol{J}_{\text {target,avoid }}\right| \leq X_{1}\left|\boldsymbol{J}_{1}^{\text {target,avoid }}\right|+X_{2}\left|\boldsymbol{J}_{2}^{\text {target,avoid }}\right| \\
& +\cdots+X_{N E}\left|\boldsymbol{J}_{N E}^{\text {target,avoid }}\right| \text { and } \\
& \text { mean }(J) \leq \operatorname{mean}\left(X_{1}\left|\boldsymbol{J}_{1}\right|\right)+\operatorname{mean}\left(X_{2}\left|\boldsymbol{J}_{2}\right|\right)+\cdots \\
& + \text { mean }\left(X_{N E}\left|\boldsymbol{J}_{N E}\right|\right) \text { or } \\
& \text { mean }\left(J_{\text {target,avoid }}\right) \leq \text { mean }\left(X_{1}\left|\boldsymbol{J}_{1}^{\text {target,avoid }}\right|\right)+\text { mean } \\
& \left(X_{2}\left|\boldsymbol{J}_{2}^{\text {target,avoid }}\right|\right)+\cdots+\text { mean }\left(X_{N E}\left|\boldsymbol{J}_{N E}^{\text {target,avoid }}\right|\right) .
\end{aligned}
$$

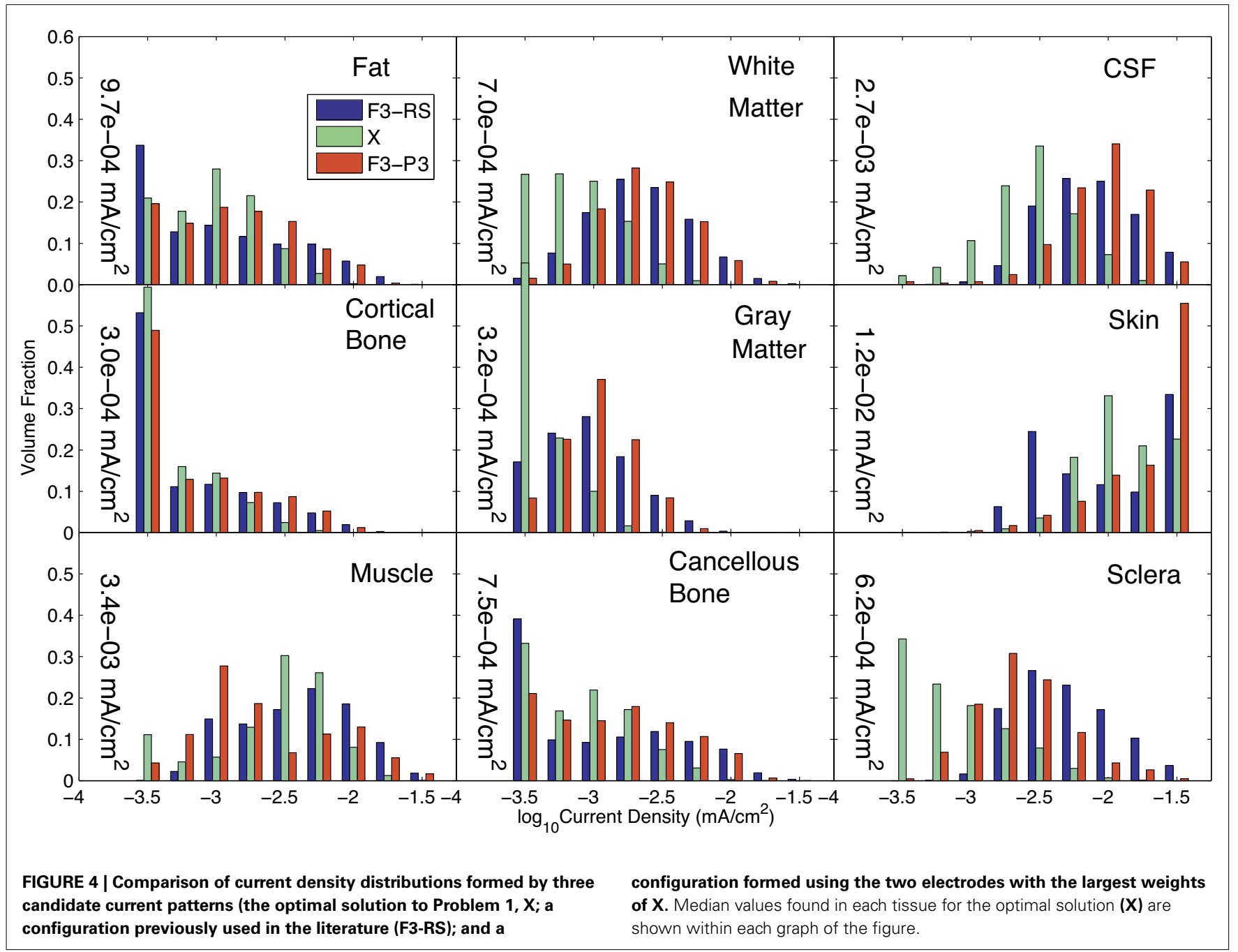


In the first step of the optimization algorithm, our precomputed gradient was compared with internal estimations of gradient and found to agree within a relative tolerance of $1 \times 10^{-6}$. Thus, we believe that the precomputed gradient provided a satisfactory estimate to guide optimization. In the results that follow, we continue to present our findings in terms of median values.

\section{PROBLEMS CONSIDERED}

We tested the optimization procedure in the context of three different problems. First, we sought to deliver current preferentially to the left IFG, while avoiding delivery to the accumbens (Problem 1 ). In this problem we required that the $J_{\max }$ experienced by left and right accumbens was less than $0.5 \mu \mathrm{A} / \mathrm{cm}^{2}$, while we chose $C_{\min }$ and $C_{\max }$ to be 0.5 and $2 \mathrm{~mA}$, respectively. We also required that the mean $J$ in the left IFG was at least twice the mean $J$ in the accumbens $(r=2)$.

In Problem 2, we wished to deliver maximal $J$ to the accumbens. No "avoid" region was nominated, but we again chose $C_{\min }$ and $C_{\max }$ to be 0.5 and $2 \mathrm{~mA}$, respectively.

In Problem 3, we again nominated the accumbens as the target, but specified that the left IFG be avoided. We set the mean $J$ ratio, $r$, to be 1 . Again, $C_{\min }$ and $C_{\max }$ were 0.5 and $2 \mathrm{~mA}$, respectively.

\section{RESULTS}

\section{PROBLEM 1}

We executed Problem 1 using the procedures outlined above and obtained a result $\mathbf{X}$ that satisfied all constraints. The optimization algorithm was terminated because the step size was smaller than the threshold value of $10^{-10}$. First-order optimality was found to be around $10^{-3}$. The values of individual coefficients are plotted in Figure 3. Note that the positive weights of each electrode were biased toward those near the left IFG, such as F3.

We compared the results of Problem 1 optimization with those achieved for an earlier simulation in which only two electrodes were used [F3 and a right supraorbital (RS) electrode]. We also computed the current densities resulting from an F3-P3 pattern, given that the estimated $\mathbf{X}$ value contained large coefficients for each of these electrodes. The results for these three configurations are compared in Figure 4, showing the current distributions in different tissues. The 1-norm of the total current found for our "optimized" problem, $C=1.15 \mathrm{~mA}$, was scaled so that the total injected current had the same value of $1 \mathrm{~mA}$ in all three configurations.

The median current density in different tissues found in each of these three configurations is shown in Table 2. The current densities in the target and avoided regions are highlighted in green and red respectively.

The current distributions in peripheral cortical tissues are summarized in Figure 5. The distributions in the IFG, DLPFC, and angular gyrus are shown bilaterally. The median current densities in the left IFG were approximately four times those in the right IFG.

\section{PROBLEM 2}

Solution of Problem 2, which sought to maximize mean current densities in the accumbens with no "avoid" region specified, was terminated because the maximum number of iterations was
Table 2 | Median current density values found in different tissues and structures for Problem 1.

\begin{tabular}{|c|c|c|c|}
\hline & $\begin{array}{l}X \\
\mathrm{~mA} / \mathrm{cm}^{2}\end{array}$ & $\begin{array}{l}\text { F3-RS } \\
\mathrm{mA} / \mathrm{cm}^{2}\end{array}$ & $\begin{array}{l}\text { F3-P3 } \\
\mathrm{mA} / \mathrm{cm}^{2}\end{array}$ \\
\hline \multicolumn{4}{|l|}{ TISSUE } \\
\hline Blood & $1.04 \times 10^{-4}$ & $4.45 \times 10^{-3}$ & $4.85 \times 10^{-4}$ \\
\hline Cancelous bone & $7.49 \times 10^{-5}$ & $8.04 \times 10^{-4}$ & $1.32 \times 10^{-4}$ \\
\hline Cortical bone & $2.97 \times 10^{-5}$ & $3.66 \times 10^{-4}$ & $4.41 \times 10^{-5}$ \\
\hline CSF & $2.75 \times 10^{-4}$ & $7.49 \times 10^{-3}$ & $9.36 \times 10^{-4}$ \\
\hline Fat & $9.67 \times 10^{-4}$ & $8.54 \times 10^{-4}$ & $1.22 \times 10^{-4}$ \\
\hline Gray matter & $3.21 \times 10^{-4}$ & $9.06 \times 10^{-4}$ & $1.01 \times 10^{-4}$ \\
\hline Muscle & $3.36 \times 10^{-3}$ & $4.52 \times 10^{-3}$ & $1.53 \times 10^{-4}$ \\
\hline Sclera & $6.22 \times 10^{-4}$ & $4.65 \times 10^{-3}$ & $2.12 \times 10^{-4}$ \\
\hline Skin & $1.18 \times 10^{-2}$ & $9.42 \times 10^{-3}$ & $2.80 \times 10^{-3}$ \\
\hline White matter & $6.98 \times 10^{-4}$ & $2.26 \times 10^{-3}$ & $2.22 \times 10^{-4}$ \\
\hline \multicolumn{4}{|c|}{ CORTICAL STRUCTURE } \\
\hline$A G(L)$ & $7.20 \times 10^{-4}$ & $9.31 \times 10^{-4}$ & $2.22 \times 10^{-3}$ \\
\hline$A G(R)$ & $3.10 \times 10^{-4}$ & $5.96 \times 10^{-4}$ & $7.00 \times 10^{-4}$ \\
\hline Cingulate & $2.88 \times 10^{-4}$ & $9.09 \times 10^{-4}$ & $1.18 \times 10^{-3}$ \\
\hline DLPFC (L) & $1.13 \times 10^{-3}$ & $1.52 \times 10^{-3}$ & $2.82 \times 10^{-3}$ \\
\hline DLPFC (R) & $3.26 \times 10^{-4}$ & $1.68 \times 10^{-3}$ & $9.78 \times 10^{-4}$ \\
\hline Frontal lobe & $4.39 \times 10^{-4}$ & $2.07 \times 10^{-3}$ & $1.31 \times 10^{-3}$ \\
\hline IFG (L) & $8.26 \times 10^{-4}$ & $1.87 \times 10^{-3}$ & $2.14 \times 10^{-3}$ \\
\hline IFG (R) & $2.63 \times 10^{-4}$ & $1.49 \times 10^{-3}$ & $7.73 \times 10^{-4}$ \\
\hline Occipital lobe & $3.14 \times 10^{-4}$ & $3.84 \times 10^{-4}$ & $8.89 \times 10^{-4}$ \\
\hline Parietal lobe & $3.92 \times 10^{-4}$ & $6.45 \times 10^{-4}$ & $1.20 \times 10^{-3}$ \\
\hline Temporal lobe & $3.49 \times 10^{-4}$ & $8.72 \times 10^{-4}$ & $8.93 \times 10^{-4}$ \\
\hline \multicolumn{4}{|c|}{ DEEP STRUCTURE } \\
\hline Accumbens & $9.74 \times 10^{-5}$ & $1.38 \times 10^{-3}$ & $9.08 \times 10^{-4}$ \\
\hline Amygdala & $2.19 \times 10^{-4}$ & $1.37 \times 10^{-3}$ & $1.18 \times 10^{-3}$ \\
\hline Caudate nucleus & $1.66 \times 10^{-4}$ & $1.70 \times 10^{-3}$ & $9.13 \times 10^{-4}$ \\
\hline Cerebellar GM & $2.03 \times 10^{-4}$ & $4.06 \times 10^{-4}$ & $4.92 \times 10^{-4}$ \\
\hline Hippocampus & $2.46 \times 10^{-4}$ & $1.15 \times 10^{-3}$ & $8.91 \times 10^{-4}$ \\
\hline Globus pallidus & $1.65 \times 10^{-4}$ & $1.20 \times 10^{-3}$ & $9.26 \times 10^{-4}$ \\
\hline Putamen & $2.06 \times 10^{-4}$ & $1.37 \times 10^{-3}$ & $8.70 \times 10^{-4}$ \\
\hline Thalamus & $2.01 \times 10^{-4}$ & $1.03 \times 10^{-3}$ & $1.02 \times 10^{-3}$ \\
\hline
\end{tabular}

The optimal weighting $\boldsymbol{X}$ was compared with another candidate pattern (F3-RS) and a pattern found using the 2 greatest weights in $\boldsymbol{X}$. Median values in targeted and avoided regions are highlighted in green and red shading, respectively.

exceeded. However, substantial progress toward a solution was made. We found that the optimization procedure produced a clear bias toward anterior and posterior electrodes. Also, there were only four electrodes with an absolute normalized weight greater than $1 \mu \mathrm{A}$ - electrodes F7, O1, O2, Oz, and T6. The electrode with the largest weight, F7, was not centrally located, being on the lower left head, and all other electrodes had negative weights. We believe that this unexpected bias may have resulted from inhomogeneity in the conductivity distribution or white matter directions. The problem resulted in a first-order optimization value of about $2 \times 10^{-3}$, larger than the value found in solving Problem 1 .

Distribution estimations within basal ganglia and peripheral cortical structures for Problem 2 are plotted in Figure 6 for the normalized optimized pattern. A comparison with a 2-electrode pattern chosen by using only the electrodes with the two largest 


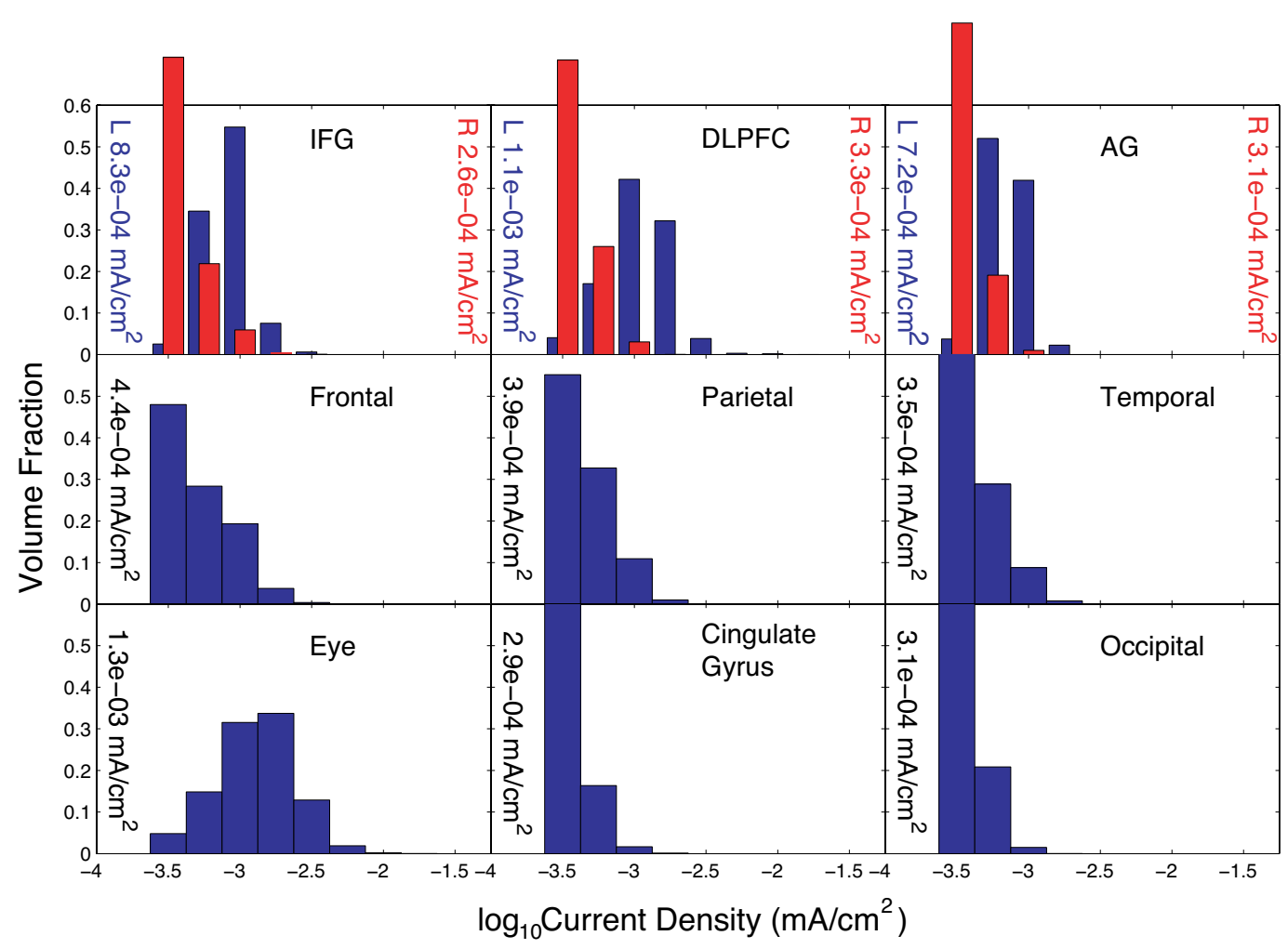

FIGURE 5 | Distribution of current densities in peripheral cortical structures in Problem 1 found using the optimal solution, X. Median values in each structure obtained using the optimal solution are shown within each graph of the figure. IFG, DLPFC, and AG refer to the inferior frontal gyrus
(IFG), dorsolateral prefrontal cortex (DLPFC), and angular gyrus (AG), respectively. For the IFG, DLPFC, and AG, current density distributions are shown separately for left (blue) and right (red) structures, with median values shown on either side of each plot. magnitude weights found by the optimization procedure, F7 and $\mathrm{Oz}$, is shown in Table 3. The current densities found in target structures by the optimization procedure (using five electrodes) were very similar to this 2-electrode pattern. Median eye current densities found for both the F7-Oz pattern and the optimal solution were around $10 \mu \mathrm{A} / \mathrm{cm}^{2}$. The threshold for phosphene generation cited in the literature $\left(8 \mathrm{~mA} / \mathrm{m}^{2}\right.$ or $0.8 \mu \mathrm{A} / \mathrm{cm}^{2}$; Reilly, 1998) was based on stimulation at $20 \mathrm{~Hz}$. Therefore, even though the threshold for DC stimulation might in fact be at least a factor of 10 higher (Adrian, 1977), we would expect this current pattern to produce phosphenes.

A test performed using the $\mathrm{F} 7-\mathrm{Oz}$ pattern as an initial point for the procedure resulted in no progress toward the final solution. Interestingly, the first-order optimality measure found using F7$\mathrm{Oz}$ was $3.5 \times 10^{-3}$, larger than that found for the final value of $\mathbf{X}$ for Problem 2, which was around $2 \times 10^{-3}$.

\section{PROBLEM 3}

The pattern found when the IFG was specified as the "avoid" region was biased toward electrodes on the right side of the head, as expected. Execution of Problem 3 was terminated because the step size decreased below threshold. Results for the normalized optimized pattern are shown in Figure 7 for peripheral and deep structures. Table 4 shows median values in different structures for this pattern and for a 2-electrode pattern found by combining the electrodes that had the two largest magnitude weights in $\mathrm{X}-\mathrm{C} 4$ and FPz. Current densities found in the right cortex were generally larger than those in the left cortex or deep brain structures. Median current densities in the eye for this case were larger than in Problem 2 (around $7 \times 10^{-2} \mathrm{~mA} / \mathrm{cm}^{2}$ ), and therefore phosphene generation would be highly likely with this configuration.

\section{USE OF FEWER THAN 19 ELECTRODES}

Results obtained by the optimization, with approximate normalized "optimal" patterns created using the 2-, 4-, and 6-highest magnitude current electrodes are shown in Table 5, now comparing target and avoid regions for each pattern. In this test, if the sum of currents from the set of electrodes was found to be non-zero (contrary to constraint 1), we assumed that remaining current flowed to an extracranial electrode. These electrode patterns resulted in distributions in the target or avoid structures being of the same magnitude as those found using the full 19-electrode montage.

\section{DISCUSSION}

The solution of problem 1 demonstrates how an optimization approach might be used to allow more efficient and precise targeting of tDCS currents to nominated brain regions and enable steering of current away from other specified areas in individual subjects. The solution we found for this problem successfully 

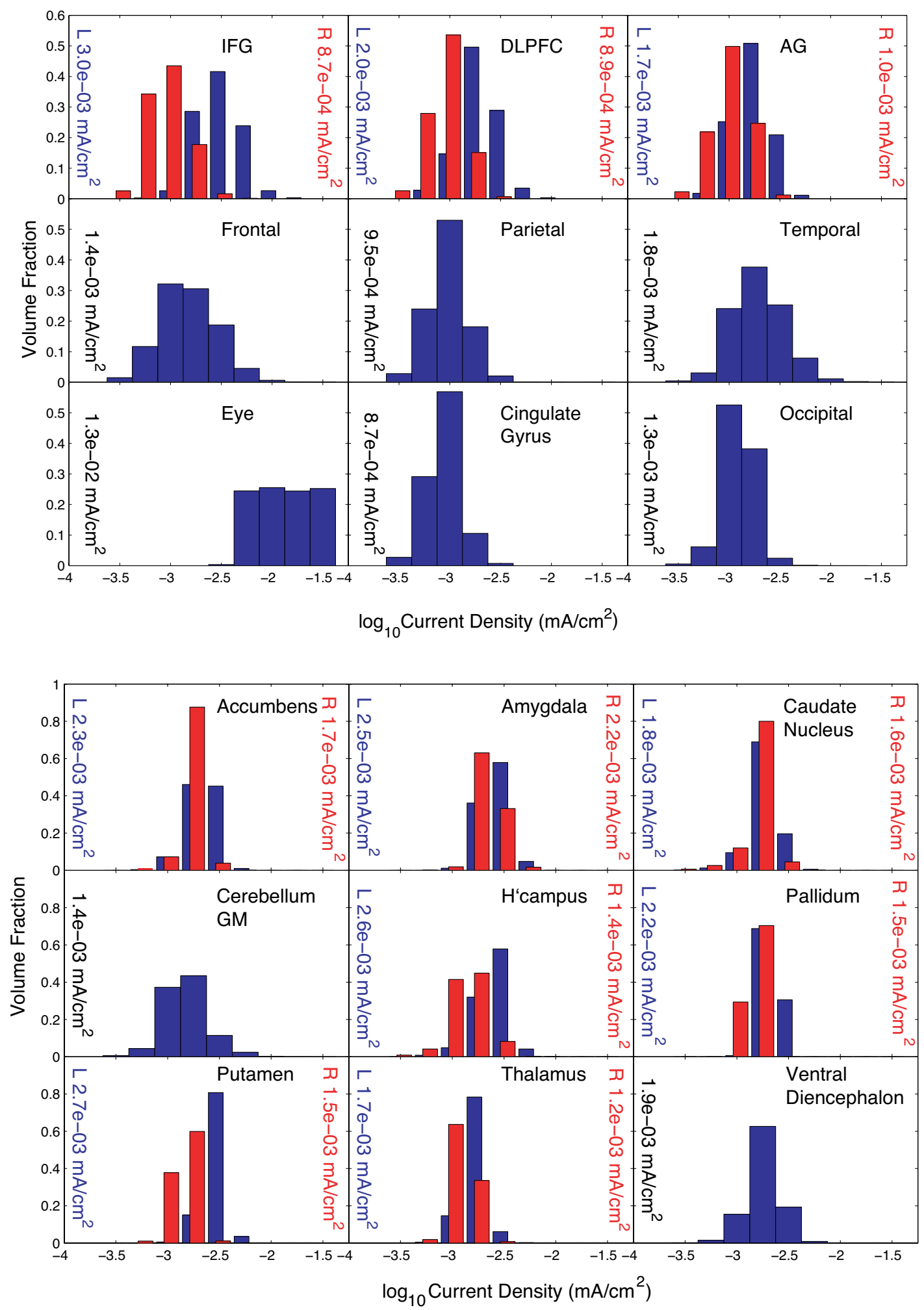

FIGURE 6 | Distribution of current densities in (top) peripheral cortical structures and (bottom) deep brain structures for Problem 2. Median values in each structure obtained using the optimal solution $\mathbf{X}$ are shown within each graph of the figure. IFG, DLPFC, and AG refer to the inferior frontal gyrus (IFG), dorsolateral prefrontal cortex (DLPFC), and angular gyrus $(A G)$, respectively. For the IFG, DLPFC, and AG, current density distributions are shown separately for left (blue) and right (red) structures, with median values shown on either side of each plot. 
directed current away from the accumbens (producing a bilateral median current density of $\left.9.74 \times 10^{-5} \mathrm{~mA} / \mathrm{cm}^{2}\right)$ and producing a median current density in the IFG of $8.26 \times 10^{-4} \mathrm{~mA} / \mathrm{cm}^{2}$ in the IFG target. By comparison, the two alternative current patterns, F3-RS and F3-P3, although producing larger current densities in the IFG, both produced median current densities in the accumbens that were at least a factor of 10 larger. The ability to selectively deliver current to different structures may therefore facilitate experiments relating to the structures and mechanisms involved in tDCS effects, particularly when implemented using subject-specific models. Further, use of distributed (i.e., more than two electrodes) current patterns may reduce skin currents and the likelihood of peripheral nerve stimulation and therefore provide a safety benefit over other patterns.

\section{USE OF FEWER ELECTRODES}

It may also be that patterns using fewer electrodes, based on these "optimal" designs, can be achieved, as demonstrated in Section "Use of Fewer Than 19 Electrodes." These patterns could be implemented by coupling several current generators together. Use of a selection of higher weighted electrodes in combination with a single extracranial electrode might provide a practical method of implementing computed patterns.

\section{SAFETY CONSIDERATIONS}

The maximal skin currents shown in Table $\mathbf{5}$ were reduced as more electrodes were incorporated. Therefore, use of more electrodes may make it possible to apply a larger total current and achieve some current steering without causing peripheral nerve stimulation. The nominal current density value thought to produce peripheral nerve stimulation is about $0.1 \mathrm{~mA} / \mathrm{cm}^{2}$ (Reilly, 1998). Note that in all but one case shown in Table 5, the predicted maximum skin current densities were above this limit. However, these current densities were observed in very small volumes near electrodes, and it is unclear whether these patterns would actually result in a subject's perception of the current. In the two problems targeting deep structures, we observed median eye currents of the order of $0.1 \mathrm{~mA} / \mathrm{cm}^{2}$. This prediction implies that phosphene generation is likely using these patterns. Use of the eye as an "avoid" region might produce more acceptable patterns.

\section{USE OF MORE CONSTRAINTS}

The problems we have considered here involve a fixed amount of current applied to the head. This current must flow somewhere. Use of "avoid" constraints may result in large currents being observed in areas that are neither avoided nor target regions, such as those found in right peripheral cortical regions in Problem 3. This issue will obviously be more prevalent as more avoided regions are chosen and will depend on the relative geometry of electrodes, avoided regions, and target regions. We expect that it may not be possible to solve some over constrained optimization tasks, or to find a feasible starting point.

A corollary finding is that these observations may be beneficial and provide alternatives to previous stimulation protocols. For example, the median $J$ found in the left IFG by Problem 2 was larger than that found using the F3-RS current pattern, which has been presumed appropriate for stimulating this area. If applying a
Table 3 | Median current density values found in different tissues and structures for Problem 2.

\begin{tabular}{|c|c|c|c|}
\hline & $\begin{array}{l}\mathrm{X} \\
\mathrm{mA} / \mathrm{cm}^{2}\end{array}$ & $\begin{array}{l}\mathrm{FPz}-\mathrm{Oz} \\
\mathrm{mA} / \mathrm{cm}^{2}\end{array}$ & $\begin{array}{l}\mathrm{F7}-\mathrm{Oz} \\
\mathrm{mA} / \mathrm{cm}^{2}\end{array}$ \\
\hline \multicolumn{4}{|l|}{ TISSUE } \\
\hline Blood & $6.00 \times 10^{-3}$ & $5.94 \times 10^{-3}$ & $6.07 \times 10^{-3}$ \\
\hline Cancelous bone & $6.89 \times 10^{-4}$ & $6.69 \times 10^{-4}$ & $6.81 \times 10^{-4}$ \\
\hline Cortical bone & $6.09 \times 10^{-4}$ & $3.42 \times 10^{-4}$ & $5.76 \times 10^{-4}$ \\
\hline CSF & $1.00 \times 10^{-2}$ & $1.21 \times 10^{-2}$ & $1.02 \times 10^{-2}$ \\
\hline Fat & $1.09 \times 10^{-3}$ & $8.46 \times 10^{-4}$ & $9.90 \times 10^{-4}$ \\
\hline Gray matter & $1.38 \times 10^{-3}$ & $1.36 \times 10^{-3}$ & $1.40 \times 10^{-3}$ \\
\hline Muscle & $6.84 \times 10^{-3}$ & $3.56 \times 10^{-3}$ & $6.78 \times 10^{-3}$ \\
\hline Sclera & $7.47 \times 10^{-3}$ & $6.58 \times 10^{-3}$ & $7.53 \times 10^{-3}$ \\
\hline Skin & $1.14 \times 10^{-2}$ & $8.38 \times 10^{-3}$ & $1.11 \times 10^{-2}$ \\
\hline White matter & $6.92 \times 10^{-3}$ & $2.81 \times 10^{-3}$ & $6.96 \times 10^{-3}$ \\
\hline \multicolumn{4}{|c|}{ CORTICAL STRUCTURE } \\
\hline$A G(L)$ & $1.69 \times 10^{-3}$ & $1.27 \times 10^{-3}$ & $1.70 \times 10^{-3}$ \\
\hline$A G(R)$ & $1.00 \times 10^{-3}$ & $1.27 \times 10^{-3}$ & $9.80 \times 10^{-4}$ \\
\hline Cingulate & $8.70 \times 10^{-4}$ & $1.07 \times 10^{-3}$ & $8.86 \times 10^{-4}$ \\
\hline DLPFC (L) & $1.99 \times 10^{-3}$ & $1.86 \times 10^{-3}$ & $2.00 \times 10^{-3}$ \\
\hline DLPFC (R) & $8.92 \times 10^{-4}$ & $1.71 \times 10^{-3}$ & $8.92 \times 10^{-4}$ \\
\hline Frontal lobe & $1.44 \times 10^{-3}$ & $1.82 \times 10^{-3}$ & $1.45 \times 10^{-3}$ \\
\hline IFG (L) & $3.03 \times 10^{-3}$ & $1.83 \times 10^{-3}$ & $3.05 \times 10^{-3}$ \\
\hline IFG (R) & $8.73 \times 10^{-4}$ & $1.62 \times 10^{-3}$ & $8.75 \times 10^{-4}$ \\
\hline Occipital lobe & $1.26 \times 10^{-3}$ & $1.12 \times 10^{-3}$ & $1.32 \times 10^{-3}$ \\
\hline Parietal lobe & $9.47 \times 10^{-4}$ & $9.72 \times 10^{-4}$ & $9.50 \times 10^{-4}$ \\
\hline Temporal lobe & $1.85 \times 10^{-3}$ & $1.44 \times 10^{-3}$ & $1.87 \times 10^{-3}$ \\
\hline \multicolumn{4}{|c|}{ DEEP STRUCTURE } \\
\hline Accumbens (L) & $2.33 \times 10^{-3}$ & $1.46 \times 10^{-3}$ & $2.34 \times 10^{-3}$ \\
\hline Accumbens (R) & $1.74 \times 10^{-3}$ & $1.42 \times 10^{-3}$ & $1.75 \times 10^{-3}$ \\
\hline Amygdala & $2.37 \times 10^{-3}$ & $1.95 \times 10^{-3}$ & $2.39 \times 10^{-3}$ \\
\hline Caudate nucleus & $1.70 \times 10^{-3}$ & $1.26 \times 10^{-3}$ & $1.71 \times 10^{-3}$ \\
\hline Cerebellar GM & $1.43 \times 10^{-3}$ & $1.40 \times 10^{-3}$ & $1.46 \times 10^{-3}$ \\
\hline Hippocampus & $2.01 \times 10^{-3}$ & $1.52 \times 10^{-3}$ & $2.03 \times 10^{-3}$ \\
\hline Globus pallidus & $1.80 \times 10^{-3}$ & $1.32 \times 10^{-3}$ & $1.82 \times 10^{-3}$ \\
\hline Putamen & $2.06 \times 10^{-3}$ & $1.23 \times 10^{-3}$ & $2.07 \times 10^{-3}$ \\
\hline Thalamus & $1.42 \times 10^{-3}$ & $1.48 \times 10^{-3}$ & $1.43 \times 10^{-3}$ \\
\hline
\end{tabular}

The optimal weighting $\boldsymbol{X}$ is compared with a symmetric pattern (FPz-Oz) and a pattern found using the 2 greatest weights in $\boldsymbol{X}$ (F7-Oz). Median values in the targeted region are highlighted in green shading.

large current to the left IFG is the only requirement, then a pattern similar to that found in Problem 2 might also be considered to stimulate the IFG of a similar subject.

\section{OPTIMALITY}

The results we have found have satisfied the requirements specified to the optimization algorithm, with some exceptions. However, there is no guarantee that the solution is a global optimum or even unique. A trivial demonstration of the non-uniqueness of solutions is that exactly the same current densities as any candidate weighting, $\mathbf{X}$, will be produced by $-\mathbf{X}$, since most constraints and objective function are based solely on current density magnitude. This lack of uniqueness could be resolved by introducing a 

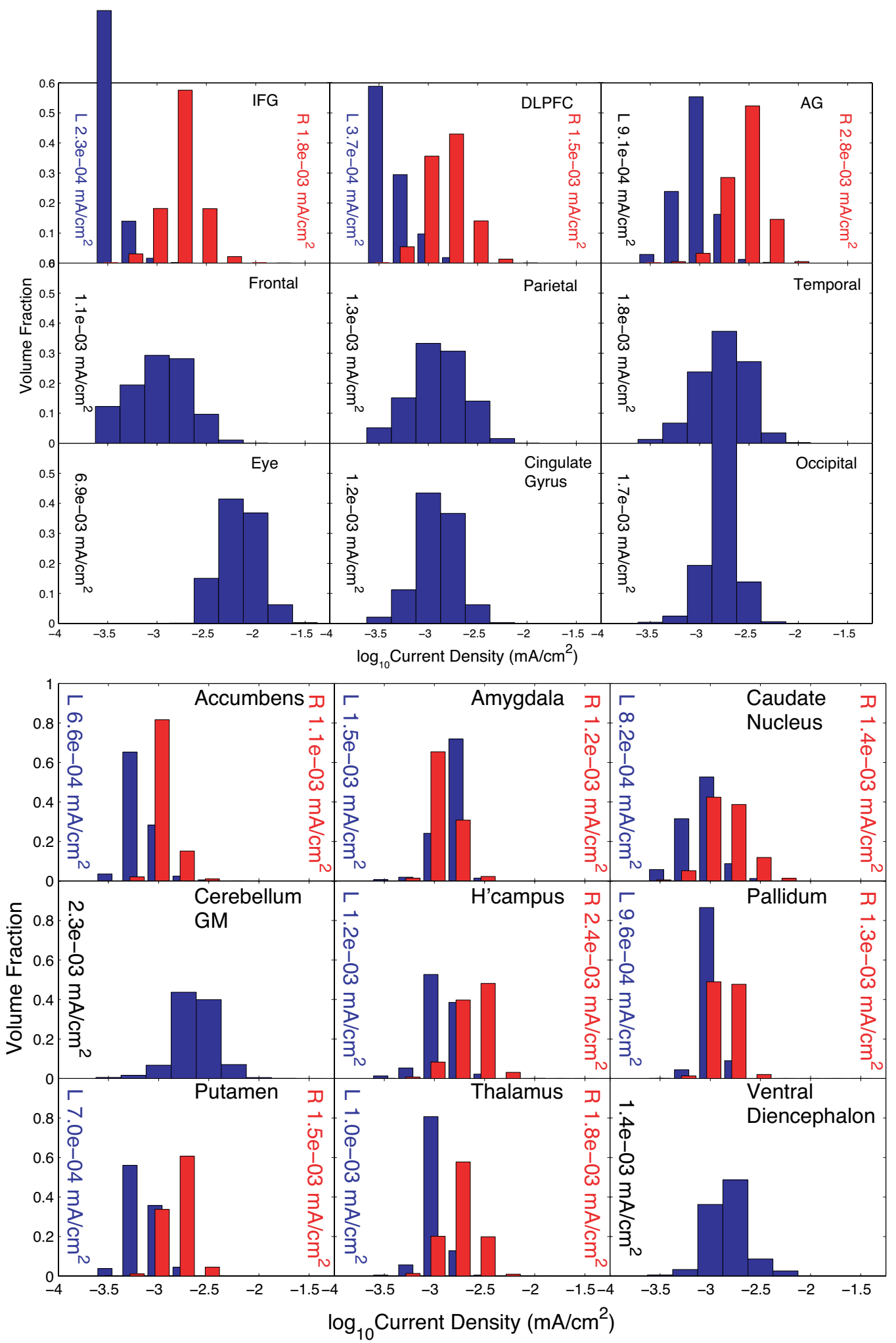

FIGURE 7 | Distribution of current densities in (top) peripheral cortical structures and (bottom) deep brain structures for Problem 3. Median values in each structure obtained using the optimal solution $\mathbf{X}$ are shown within each graph of the figure. IFG, DLPFC, and AG refer to the inferior frontal gyrus (IFG), dorsolateral prefrontal cortex (DLPFC), and angular gyrus $(A G)$, respectively. For the IFG, DLPFC, and AG, current density distributions are shown separately for left (blue) and right (red) structures, with median values shown on either side of each plot. 
Table 4 | Median current density values found in different tissues and structures for Problem 3.

\begin{tabular}{|c|c|c|}
\hline & $\begin{array}{l}\mathrm{X} \\
\mathrm{mA} / \mathrm{cm}^{2}\end{array}$ & $\begin{array}{l}\mathrm{C} 4-\mathrm{FPz} \\
\mathrm{mA} / \mathrm{cm}^{2}\end{array}$ \\
\hline \multicolumn{3}{|l|}{ TISSUE } \\
\hline Blood & $5.31 \times 10^{-4}$ & $4.67 \times 10^{-3}$ \\
\hline Cancelous bone & $1.66 \times 10^{-3}$ & $1.31 \times 10^{-3}$ \\
\hline Cortical bone & $7.18 \times 10^{-4}$ & $3.42 \times 10^{-4}$ \\
\hline CSF & $1.00 \times 10^{-2}$ & $9.91 \times 10^{-3}$ \\
\hline Fat & $2.41 \times 10^{-3}$ & $1.25 \times 10^{-3}$ \\
\hline Gray matter & $1.48 \times 10^{-3}$ & $1.09 \times 10^{-3}$ \\
\hline Muscle & $7.79 \times 10^{-3}$ & $3.01 \times 10^{-3}$ \\
\hline Sclera & $2.90 \times 10^{-3}$ & $4.93 \times 10^{-3}$ \\
\hline Skin & $2.46 \times 10^{-2}$ & $1.58 \times 10^{-2}$ \\
\hline White matter & $3.09 \times 10^{-3}$ & $2.75 \times 10^{-3}$ \\
\hline \multicolumn{3}{|c|}{ CORTICAL STRUCTURE } \\
\hline$A G(L)$ & $9.10 \times 10^{-4}$ & $5.48 \times 10^{-4}$ \\
\hline$A G(R)$ & $2.85 \times 10^{-3}$ & $2.55 \times 10^{-3}$ \\
\hline Cingulate & $1.24 \times 10^{-3}$ & $1.36 \times 10^{-3}$ \\
\hline DLPFC (L) & $3.67 \times 10^{-4}$ & $1.67 \times 10^{-3}$ \\
\hline DLPFC (R) & $1.46 \times 10^{-3}$ & $2.82 \times 10^{-3}$ \\
\hline Frontal lobe & $6.89 \times 10^{-3}$ & $1.94 \times 10^{-3}$ \\
\hline IFG (L) & $2.28 \times 10^{-4}$ & $1.43 \times 10^{-3}$ \\
\hline IFG (R) & $1.76 \times 10^{-3}$ & $2.11 \times 10^{-3}$ \\
\hline Occipital lobe & $1.69 \times 10^{-3}$ & $6.92 \times 10^{-4}$ \\
\hline Parietal lobe & $1.26 \times 10^{-3}$ & $1.03 \times 10^{-3}$ \\
\hline Temporal lobe & $1.79 \times 10^{-3}$ & $7.06 \times 10^{-4}$ \\
\hline \multicolumn{3}{|c|}{ DEEP STRUCTURE } \\
\hline Accumbens (L) & $6.56 \times 10^{-4}$ & $1.00 \times 10^{-3}$ \\
\hline Accumbens (R) & $1.11 \times 10^{-3}$ & $1.36 \times 10^{-4}$ \\
\hline Amygdala & $1.37 \times 10^{-3}$ & $1.19 \times 10^{-3}$ \\
\hline Caudate nucleus & $1.04 \times 10^{-3}$ & $1.12 \times 10^{-3}$ \\
\hline Cerebellum GM & $2.31 \times 10^{-3}$ & $7.04 \times 10^{-4}$ \\
\hline Hippocampus & $1.57 \times 10^{-3}$ & $7.85 \times 10^{-4}$ \\
\hline Globus pallidus & $1.16 \times 10^{-3}$ & $8.97 \times 10^{-4}$ \\
\hline Putamen & $1.12 \times 10^{-3}$ & $9.93 \times 10^{-4}$ \\
\hline Thalamus & $1.27 \times 10^{-3}$ & $9.91 \times 10^{-4}$ \\
\hline
\end{tabular}

The optimal weighting $\boldsymbol{X}$ is compared with a pattern found using the 2 greatest weights in $X(C 4-P z)$. Median values in targeted and avoided regions are highlighted in green and red shading, respectively.

constraint on a single electrode, $i$, that restricted its coefficient, $X_{\mathrm{i}}$, to be either less than or greater than zero.

The optimality measure produced by the algorithm, a numerical measure of the gradient of the objective function at each iteration, was found to be less than $10^{-3}$ for solution of Problem 1 . We know that our gradient estimation is not exact, but this value should provide some indication of the landscape of the objective function. Even if gradient estimation is exact, finding an optimality measure that suggests the objective function is at or near an extreme value does not guarantee that the solution attained is a global minimum.

Solutions in Problems 2 and 3 produced optimality measures of around 2 and $3 \times 10^{-3}$, respectively. Solutions in these two problems took many more iterations to produce than in Problem
Table 5 | Comparison of effects of using 2-, 4- and 6-electrode patterns based on optimal current patterns.

\begin{tabular}{|c|c|c|c|c|}
\hline & $\begin{array}{l}\mathrm{X} 2 \\
\mathrm{~mA} / \mathrm{cm}^{2}\end{array}$ & $\begin{array}{l}\mathrm{X} 4 \\
\mathrm{~mA} / \mathrm{cm}^{2}\end{array}$ & $\begin{array}{l}\mathrm{X} 6 \\
\mathrm{~mA} / \mathrm{cm}^{2}\end{array}$ & $\begin{array}{l}\mathrm{X} 19 \\
\mathrm{~mA} / \mathrm{cm}^{2}\end{array}$ \\
\hline \multicolumn{5}{|l|}{ PROBLEM 1} \\
\hline$F G(L)$ & $2.19 \times 10^{-3}$ & $1.77 \times 10^{-3}$ & $1.46 \times 10^{-3}$ & $8.26 \times 10^{-4}$ \\
\hline$-G(R)$ & $1.25 \times 10^{-3}$ & $1.33 \times 10^{-3}$ & $1.24 \times 10^{-3}$ & $2.63 \times 10^{-4}$ \\
\hline ccumbens (L) & $1.55 \times 10^{-3}$ & $1.41 \times 10^{-3}$ & $1.22 \times 10^{-3}$ & $9.74 \times 10^{-5}$ \\
\hline 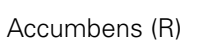 & $1.41 \times 10^{-3}$ & $1.31 \times 10^{-3}$ & $1.13 \times 10^{-3}$ & $2.19 \times 10^{-4}$ \\
\hline kin maximum & $3.17 \times 10^{-1}$ & $2.24 \times 10^{-1}$ & $1.80 \times 10^{-1}$ & $10^{-1}$ \\
\hline \multicolumn{5}{|l|}{ PROBLEM 2} \\
\hline $\mathrm{FG}(\mathrm{L})$ & $\times 10^{-3}$ & $.05 \times 10^{-3}$ & - & $3 \times 10^{-3}$ \\
\hline $\mathrm{G}(\mathrm{R})$ & $9.06 \times 10^{-4}$ & $8.75 \times 10^{-4}$ & - & -4 \\
\hline ccumbens (L) & $2.35 \times 10^{-3}$ & $2.34 \times 10^{-3}$ & - & $2.33 \times 10^{-3}$ \\
\hline$s(R)$ & $1.77 \times 10^{-3}$ & $1.75 \times 10^{-3}$ & - & $1.74 \times 10^{-3}$ \\
\hline um & $4.26 \times 10^{-1}$ & $4.24 \times 10^{-1}$ & - & \\
\hline \multicolumn{5}{|l|}{ PROBLEM 3} \\
\hline$=G(L)$ & $\times 10^{-4}$ & $9.50 \times 10^{-4}$ & $6.97 \times 10^{-4}$ & $2.28 \times 10^{-4}$ \\
\hline E $(R)$ & $1.91 \times 10^{-3}$ & $1.38 \times 10^{-3}$ & $1.03 \times 10^{-3}$ & $1.76 \times 10^{-3}$ \\
\hline ccumb & $1.15 \times 10^{-3}$ & $9.96 \times 10^{-4}$ & $7.59 \times 10^{-4}$ & \\
\hline ccumbens (R) & $1.23 \times 10^{-3}$ & $9.90 \times 10^{-4}$ & $7.53 \times 10^{-4}$ & $1.11 \times 10^{-4}$ \\
\hline kin maximum & $2.34 \times 10^{-1}$ & $1.32 \times 10^{-1}$ & $9.71 \times 10^{-2}$ & $2.31 \times 10^{-1}$ \\
\hline \multicolumn{5}{|c|}{$\begin{array}{l}\text { ighlighted in green and red shading } \\
\text { s are also shown for each pattern. }\end{array}$} \\
\hline \multicolumn{5}{|c|}{$\begin{array}{l}\text { 1, and solution of Problem } 2 \text { was terminated because the algorithn } \\
\text { required more than } 100 \text { iterations. Very similar results to the opti } \\
\text { mal solution }(\mathbf{X}) \text { to Problem } 2 \text { were found using its two principa } \\
\text { electrodes, and, in fact, } J \text { values in the target structure were slightl } \\
\text { larger when the two principal electrodes were used. It is possibl } \\
\text { that the F7-Oz solution is very close to the optimum solutior } \\
\text { for this Problem, and with this subject model. This finding may } \\
\text { also suggest that solutions targeting of deep structures may no } \\
\text { be unique, and that there are other possible configurations tha } \\
\text { satisfy the problem specification. }\end{array}$} \\
\hline
\end{tabular}

\section{CONCLUSION}

We demonstrated that use of a finite element model of the head, in conjunction with a non-linear optimization procedure, could result in current steering both away from and toward different structures. We found that it was possible to direct current to the left IFG while avoiding the accumbens region; to target current on the basal ganglia exclusively; and to avoid the left IFG while targeting basal ganglia. When deep structures were targeted, it was not possible to avoid delivering current to peripheral cortical regions. Further, use of this methodology revealed asymmetry in structures that may not have easily been found using other strategies. We believe that this or a similar method of optimization may prove useful in further studies of tDCS.

\section{ACKNOWLEDGMENTS}

This work was supported by the Therapeutic Cognitive Neuroscience Fund (Barry Gordon) and by the Benjamin A. Miller and Family Endowment for Aging, Alzheimer's Disease, and Autism (Barry Gordon). 


\section{REFERENCES}

Adrian, D. J. (1977). Auditory and visual sensations stimulated by lowfrequency electric currents. Radio Sci. 12, 243-250.

Akhtari, M., Bryant, H. C., Mamelak, A. N., Flynn, E. R., Heller, L., Shih, J. J., et al. (2002). Conductivities of threelayer live human skull. Brain Topogr. 14, 151-167.

Akhtari, M., Bryant, H. C., Mamelak, A. N., Heller, L., Shih, J. J., Mandelkern, M., et al. (2000). Conductivities of three-layer human skull. Brain Topogr. 13, 29-42.

Baumann, S. B., Wozny, D. R., Kelly, S. K., and Meno, F. M. (1997). The electrical conductivity of human cerebrospinal fluid at body temperature. IEEE Trans. Biomed. Eng. 44, 220-223.

Bonnans, J. F., Gilbert, J. C., Lemarechal, C., and Sagastizabal, C. (2006). Numerical Optimization - Theoretical and Practical Aspects, 2nd Edn. Berlin: Springer Verlag.

Brunelin, J., Mondino, M., Gassab, L., Haesebaert, F., Gaha, L., SuaudChagny, M., et al. (2012). Examining transcranial direct-current stimulation (tDCS) as a treatment for hallucinations in schizophrenia. Am. J. Psychiatry 169, 719-724.

Brunoni, A., Nitsche, M. A., Bolognini, N., Bikson, M., Wagner, T., Merabet, L., et al. (2012). Clinical research with transcranial direct current stimulation (tDCS): challenges and future directions. Brain Stimul. 5, 175-195.

Cogiamanian, F., Marceglia, S., Ardolino, G., Barbieri, S., and Priori, A. (2007). Improved isometric force endurance after transcranial direct current stimulation over the human motor cortical areas. Eur. J. Neurosci. 26, 242-249.

Datta, A., Bansal, V., Diaz, J., Patel, J., Reato, D., and Bikson, M. (2009). Gyri-precise head model of transcranial direct current stimulation: improved spatial focality using a ring electrode versus conventional rectangular pad. Brain Stimulat. 2, 201-207.

Dmochowski, J. P., Datta, A., Bikson, M., Su, Y., and Parra, L. C. (2011). Optimized multi-electrode stimulation increases focality and intensity at target. J. Neural Eng. 8, 046011.

Elmer, S., Burkard, M., Renz, B., Meyer, M., and Jancke, L. (2009). Direct current induced short-term modulation of the left dorsolateral prefrontal cortex while learning auditory presented nouns. Behav. Brain Funct. 5, 29.

Fregni, F., Boggio, P. S., Nitsche, M. A., Bermpohl, F., Antal, A., Feredoes, E., et al. (2005). Anodal transcranial direct current stimulation of prefrontal cortex enhances working memory. Exp. Brain Res. 166, 23-30.

Gabriel, C., Gabriel, S., and Corthout, E. (1996). The dielectric properties of biological tissues: I. Literature survey. Phys. Med. Biol. 41, 2231-2249.

Geddes, L., and Baker, L. E. (1967). The specific resistance of biological materials: a compendium of data for the biomedical engineer and physiologist. Med. Biol. Eng. Comput. 5, 271-293.

Holdefer, R. N., Sadleir, R. J., and Russell, M. J. (2006). Predicted current densities in the brain during transcranial electrical stimulation. Clin. Neurophysiol. 117, 1388-1397.

Im, C.-H., Jung, H.-H., Choi, J.-D., Lee, S. Y., and Jung, K.-Y. (2008). Determination of optimal electrode positions for transcranial direct current stimulation (tDCS). Phys. Med. Biol. 53, N219-N225.

Kirkpatrick, S., Gelatt, C. D., and Vecchi, M. P. (1983). Optimization by simulated annealing. Science 220, 671-680.

Limpert, E., Stahel, W. A., and Abbt, M. (2001). Log-normal distributions across the sciences: keys and clues. Bioscience 51, 341-352.
Loo, C. K., Alonzo, A., Martin, D., Mitchell, P. B., Galvez, V., and Sachdev, P. (2012). Transcranial direct current stimulation for depression: 3-week, randomised, sham-controlled trial. $\mathrm{Br}$. J. Psychiatry 200, 52-59.

Monti, A., Cogiamanian, F., Marceglia, S., Ferrucci, R., Marmeli, F., MrackicSposta, S., et al. (2008). Improved naming after transcranial direct current stimulation in aphasia. J. Neurol. Neurosurg. Psychiatr. 79, 451-453.

Nitsche, M. A., Cohen, L. G., Wassermann, E. M., Priori, A., Lang, N. Antal, A., et al. (2008). Transcranial direct current stimulation: state of the art 2008. Brain Stimulat. 1, 206-223.

Nitsche, M. A., and Paulus, W. (2000). Excitability changes induced in the human motor cortex by weak transcranial direct current stimulation. $J$. Physiol. 527, 633-639.

Park, J. H., Hong, S. B., Kim, D.-W., Suh, M., and Im, C.-H. (2011). A novel array-ype transcranial direct current stimulation (tDCS) system for accurate focusing on targeted brain areas. IEEE Trans. Magn. 47, 882-885.

Priori, A., Mameli, F., Cogiamanian, F. Marceglia, S., Tiriticco, M., MrackicSposta, S., et al. (2008). Lie-specific involvement of dorsolateral prefrontal cortex in deception. Cereb. Cortex 18, 451-455.

Reilly, J. P. (1998). Applied Bioelectricity: From Electrical Stimulation to Electropathology. New York: Springer.

Rubin, M., and Safdieh, J. E. (2007). Netter's Concise Neuroanatomy. Philadelphia: Saunders Elsevier.

Sadleir, R. J., and Argibay, A. (2007). Modeling skull electrical properties. Ann. Biomed. Eng. 35, 1699-1712.

Sadleir, R. J., Vannorsdall, T. D., Schretlen, D. J., and Gordon, B. (2010). Transcranial direct current stimulation (tDCS) in a realistic head model. Neuroimage 51, 1310-1318.
Scott, G. C., Joy, M. L. G., Armstrong, R. L., and Henkelman, R. M. (1991). Measurement of nonuniform current density by magnetic resonance. IEEE Trans. Med. Imaging $10,362-374$

Wagner, T., Fregni, F., Fecteau, S., Grodzinsky, A., Zahn, M., and Pascual-Leone, A. (2007). Transcranial direct current stimulation: a computer-based human model study. Neuroimage 35, 1113-1124.

Wakana, S., Jiang, H., Nagae-Poetscher, L. M., Van Zijl, P. C. M., and Mori, S. (2004). Fiber tract-based atlas of human white matter anatomy. Radiology 230, 77-87.

Waltz, R. A., Morales, J. L., Nocedal, J., and Orban, D. (2006). An interior algorithm for nonlinear optimization that combines line search and trust region steps. Math. Programming 107, 391-408.

Conflict of Interest Statement: The authors declare that the research was conducted in the absence of any commercial or financial relationships that could be construed as a potential conflict of interest.

Received: 05 July 2012; accepted: 29 September 2012; published online: 17 October 2012.

Citation: Sadleir RJ, Vannorsdall TD, Schretlen DJ and Gordon B (2012) Target optimization in transcranial direct current stimulation. Front. Psychiatry 3:90. doi: 10.3389/fpsyt.2012.00090

This article was submitted to Frontiers in Neuropsychiatric Imaging and Stimulation, a specialty of Frontiers in Psychiatry. Copyright (C) 2012 Sadleir, Vannorsdall, Schretlen and Gordon. This is an openaccess article distributed under the terms of the Creative Commons Attribution License, which permits use, distribution and reproduction in other forums, provided the original authors and source are credited and subject to any copyright notices concerning any third-party graphics etc. 